\title{
Bilateral Choroidal Neovascularization in Doyne Honeycomb Drusen
}

\section{Sumit Kumar*, Lagan Paul, Shalin Shah, Manisha Agarwal and Aditi Singal}

Vitreo-Retina, Dr Shroff Charity Eye Hospital, New Delhi, India

*Corresponding Author: Sumit Kumar, Consultant, Vitreo-Retina Department, Dr Shroff Charity Eye Hospital, New Delhi, India.
Received: April 20, 2020

Published: June 01, 2020

(C) All rights are reserved by Sumit Kumar., et al.

\begin{abstract}
A 36 year female presented with bilateral Familial Dominant drusen (Doyne Honeycomb drusen) with active choroidal neovascularization membrane (CNVM) in right eye and scarred CNVM in left eye.

Keywords: Familial Dominant Drusen; Doyne Honeycomb Drusen; CNVM; OCTA; OCT
\end{abstract}

\section{Abbreviations}

CNVM: Choroidal Neovascularization Membrane; OCTA: Optical Coherence Tomography Angiography; OCT: Optical Coherence Tomography

\section{Introduction}

A 36-year-old female presented with diminution of vision in both the eyes for last 1 month. Diminution of vision was insidious in onset, painless and gradually progressive in nature. The best corrected visual acuity was 6/18, N 18 in both the eyes. Anterior segment examination was within normal limits. Posterior segment examination showed drusens in both the eyes with a choroidal neovascular membrane (CNVM) in the right eye and a scarred CNVM in the left eye. Optical coherence tomography (OCT) scan showed extensive hyperreflective thickening beneath the retinal pigment epithelium in both the eyes with subretinal fluid collection in the right eye suggestive of active CNVM (Figure 1). Fluorescein angiography showed hyperfluoresence due to staining of the drusens at the macula and around optic nerve in both the eyes with stippled hyperfluoresence of an occult CNVM in the right eye (Figure 2A and 2B). OCTA showed immature vessels in right and mature vessels in the left eye (Figure 2C and 2D). A diagnosis of Familial Dominant Drusen with CNVM was made in both the eyes.

\section{Discussion and Conclusion}

Familial dominant drusen also known as Doyne honeycomb retinal dystrophy was first reported by Doyne in 1899 [1-3]. Fundus examination shows radiating distribution of large confluent

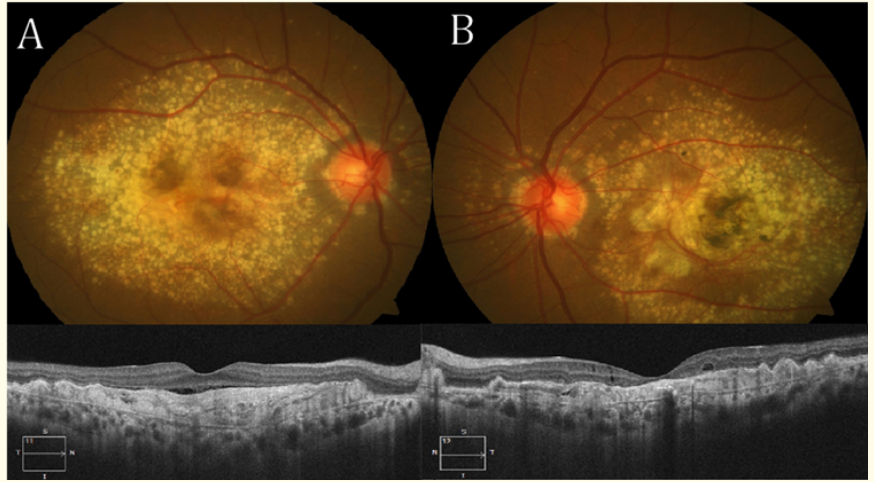

Figure 1: A: Colour fundus photograph suggestive of right eye Drusen with active CNVM and corresponding OCT suggestive of extensive hyperreflective thickening beneath the retinal pigment epithelium with subretinal fluid collection. B: Colour fundus photograph suggestive of left eye Drusen with scarred CNVM and corresponding OCT suggestive of extensive hyperreflective thickening beneath the retinal pigment epithelium.

drusens from the fovea and may also be accompanied by juxtapapillary drusens, as present in our case. Typically, the visual symptoms start at the age of 30 - 50 years comprising of reduced central vision, photophobia, and metamorphopsia [4].

And as the age progress, majority of the patients show loss of central vision and absolute scotomas develops. It can be also be complicated with development of extensive pigmentary changes, 


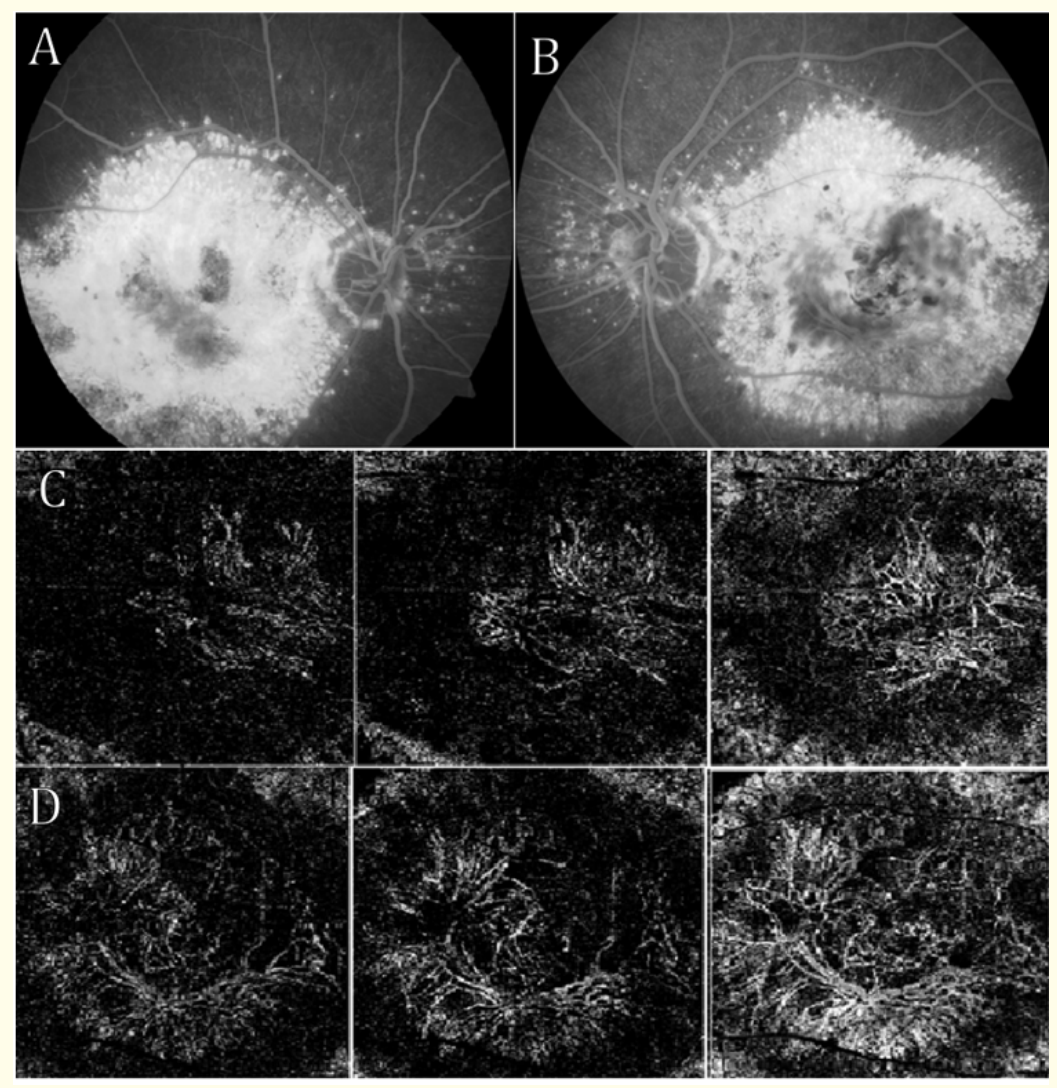

Figure 2: A and B: Fluorescein angiography showed hyperfluoresence due to staining of the drusens at the macula and around optic nerve in both the eyes with stippled hyperfluoresence of an occult CNVM in the right eye. C: OCTA image of right eye suggestive of immature vessels on outer retinal choriocapillary level. D: OCTA of left eye suggestive of mature vessels on outer retinal choriocapillary layer.

geographic atrophy (GA) or choroidal neovascularization in the region of the confluent macular drusens [4]. Our patient showed bilateral CNVM associated with familial drusens which is a rare presentation.

Extensive literature search showed three anecdotal case reports of familial drusen with CNVM [5-7]. We report this case to highlight the fact that familial drusen may be complicated with a CNVM, often likely to be missed amidst the large confluent drusen. Unlike AMD patients, these are young patients developing CNVM and need to be counselled for the symptoms of metamorphopsia and regular monitoring on an Amsler grid.

\section{Conflict of Interest}

No financial interest or any conflict of interest exists.

\section{Bibliography}

1. Pearce WG. "Doyne's honeycomb retinal degeneration. Clinical and genetic features". British Journal of Ophthalmology 52.2 (1968): 73-78.
2. Vogt A. Handbuch der gesammten Augenheilkunde. Untersuchungsmethoden. 3. Berlin: Springer Verlag (1925).

3. Heon E., et al. "Linkage of autosomal dominant radial drusen (malattia leventinese) to chromosome 2p16-21". Archives of Ophthalmology (Chicago, Ill: 1960) 114.2 (1996): 193-198.

4. Haimovici R., et al. "Symptomatic abnormalities of dark adaptation in patients with EFEMP1 retinal dystrophy (Malattia Leventinese/Doyne honeycomb retinal dystrophy)". Eye (London, England) 16.1 (2002): 7-15.

5. Anusha Pawar., et al. "Familial Dominant Drusen with Cnvm: A Case Report". Journal of Medical Science and Clinical Research 5.8 (2017):26300-26302.

6. Pager CK., et al. "Malattia leventinese presenting with subretinal neovascular membrane and hemorrhage". American Journal of Ophthalmology 131.4 (2001): 517-518. 
7. Michael Ehrlich. "Wills Eye Resident Case Series". Review of Ophthalmology (2017).

Assets from publication with us

- Prompt Acknowledgement after receiving the article

- Thorough Double blinded peer review

- Rapid Publication

- Issue of Publication Certificate

- High visibility of your Published work

Website: www.actascientific.com/

Submit Article: www.actascientific.com/submission.php

Email us: editor@actascientific.com

Contact us: +919182824667 